Proceedings of the 2011 Winter Simulation Conference

S. Jain, R.R. Creasey, J. Himmelspach, K.P. White, and M. Fu, eds.

\title{
USING SIMPLIFIED DISCRETE-EVENT SIMULATION MODELS FOR HEALTH CARE APPLICATIONS
}

\author{
Anthony Virtue \\ John Kelly \\ EC Harris LLP \\ ECHQ, 34 York Way \\ London, N1 9AB, UK
}

\author{
Thierry Chaussalet \\ University of Westminster \\ 115 Cavendish Street \\ London, W1W 6UW, UK
}

\begin{abstract}
Simulation modeling has been around for many years and produced many papers. Arguably, there has been a lack of impact in the health arena, some may say due to modeling such a large, complex, diverse and often interconnected industry. Other observations suggests that academics get rewarded for publishing large complicated models with detailed analysis rather than focusing on the requirements of the environment or the needs of implementation. This paper attempts to add to the modeling debate by suggesting that average simulation process times can act as estimators for real length of stay. This paper will also illustrate how average process time models could be used to help reconfigure emergency care services models. Average time simulation models have the potential to make a valuable contribution to modeling and they support simplified, transparent models with shortened development time.
\end{abstract}

\section{INTRODUCTION}

This paper will show that average simulation process times can act as estimators for real length of stay in health care environments. Using average time can help to simplify models, make them more transparent and reduce modeling development time. This paper will use real, readily available A\&E data to develop these simplified models. Using a stepped approached, this paper will describe how using real local A\&E data can be used to develop simplified models in a shortened development time. These simplified models, clearly linked to real local data, can help stakeholders to open up 'black boxes' and get a better understanding of high level interactions of their health care system. The data will also be used to illustrate how average time simulation models might be used to help reconfigure emergency care services and provide modeling insights into those services. These simplified models could be of great benefit as the author's experience is that stakeholders often desire models with a short turnaround time. These models can be used to provide quick illustrations of new scenarios, if required leading to more intensive investigation/modeling.

Simulation modeling has been around for many years and many papers have been produced covering a range of applications in health care. Health related simulation modeling papers include modeling geriatric length of stay by El-Darzi et al. (1998) and Vasilakis and Marshall (2005). Discrete-event simulation (DES) modeling directly related to health care includes: modeling of nurses and nursing cost in an intensive care unit (Griffiths et al. 2005); evaluation of strategies for prevention of mother-to-child HIV transmission (Rauner et al. 2005); study of an NHS walk-in centre (Ashton et al. 2005); and use of simulation to improve the blood supply chain (Katsaliaki and Brailsford 2007). More specifically, work linking DES to emergency departments includes a study combining data mining and DES for a value-added view of a hospital emergency department (Ceglowski et al. 2007) and understanding emergency department per- 


\section{Virtue, Chaussalet, and Kelly}

formance using simulation (Günal and Pidd 2006). Whilst Fletcher et al. (2007) developed a national generic A\&E model and discussed the application of its use at a local level.

Despite the number of simulations papers produced there has been a relative lack of 'real world' involvement and an even greater lack of evidence for 'real world' benefit (Taylor et al. 2009). One reason may be that health care delivery is a complex process and employs a huge number of employees delivering health care over a wide range of services. Harper and Pitt (2004) highlighted a number of issues and challenges particular to health care modeling including: scale, complexity and changes (demographic change, social and behavioral change, organizational change, political change, strategic change, technological and clinical change); diversity; buy-in and credibility; conflicting objectives; and data issues. Another observation noted by Taylor et al. was that academics are rewarded for publishing in high-quality journals and that real world links are either implicitly understood or not required as research built on previous work. In doing so, Taylor et al. suggested that researchers became disengaged from real world issues. Similarly Proudlove et al. (2007) observed that much of the published work was conducted by academics and their performance measured value large and complicated models with detailed statistical analysis of results rather focusing on the requirements of the environment and the needs of the people who need to implement changes. Günal and Pidd (2010) highlighted the extent to which DES models are used for real decisions is rarely discussed and that stake-holders need to be convinced of the benefits.

Specifically with regards to poor modeling adoption in hospitals, Sinreich and Marmor (2004) suggested the reluctance of hospital management to accept change, especially if suggestions come from a black box. To help improve acceptance of modeling in health care Sanchez et al. (2004) suggested that simulation professionals in health care needed to improve their personal capabilities to: make valid verified models; better understand their customer's business needs; and to provide customers with answers and insights to their business. Barnes et al. (1997) suggested three key elements to successful simulation in health care were: Communication and participation; User-friendly simulation software; and using simulation as a decision making tool.

This paper will use conventional A\&E data and illustrate how this service could be reconfigured into an Urgent Care Centre (UCC). The developed model will show examples how patients might be grouped to model patient pathways. The ability to model specific patient pathways, such as adult emergency treatment, adult minor treatment, elderly treatment and paediatric treatment could have direct inputs into health care planning processes and provide valuable real world information to health care planners and their clients. These models will also be able to provide valuable insights into utilization of treatment area, queues and length of stay. Although the models described in this paper are focused on emergency care, opportunities present themselves to develop similar models in other health care environments.

The models developed are modular by design, supporting rapid development for new applications. These models would have relatively low levels of abstraction as defined by Sinreich and Marmor (2004). Sinreich and Marmor described low levels of abstraction as models designed for specific systems and models that are simple and easy to use after a quick explanation. Their simplicity supports rapid feedback, early process reviews and discussions - supporting Bowers et al. (2009) observation that the development phase is the biggest benefit of simulation modeling. Lower levels of abstraction marks a move away from generic models as described by Codrington-Virtue et al. (2005b), Codrington-Virtue et al. (2005a), Günal and Pidd and Fletcher et al. (2007). Lower levels of abstraction were also applied to model patient groupings in contrast to clustered patients by diagnosis groups by Codrington-Virtue et al. (2006). Low levels of abstraction and the application of readily available data to drive the model helped to make these developed models easier to understand for the health community, minimizing objections to black box models as referenced by Sinreich and Marmor (2004).

The remainder of this paper is outlined as follows. Section 2 provides an overview of the health care planning process, A\&Es and Urgent Care Centres. Section 3 will outline the staged approach used to develop the model where stage 1 showed how real data was used to group patients (by acuity and type) and define their arrival patterns. Stage 2 determined resources' using patient group activity and stage 3 describes running and calibration of the model by comparing errors in length of stay between real and mod- 
eled patient groups with adjusted process times if applicable whilst stage 4 describes model validation and verification. Sections 4 follows with a discussion on the findings and Section 5 outlines limitations and further work in context of this paper.

\section{HEALTH CARE PLANNING AND EMERGENCY CARE PROCESSES}

\subsection{Health care planning}

Health care planning is often used to determine the size and layout of health care facility. The health care planning process function might include demand and capacity planning to: determine the correct number and size of rooms or treatment areas; assess admissions avoidance, length of stay reductions, demographic and planned services changes; and design models of care, schedules of accommodation, room layout and design to optimize patient flow and movement through clinical areas. Health care planning often work closely with estate planners as well as other managers to help facilitate user groups and stakeholder meetings. Health care planners also often consider patient types and patient groupings to design appropriate pathways. In England, health care planners often work in accordance with building guidance documents known as Health Building Notes (HBNs). Often accumulated data is used to determine capacity and demand, for example, average process time, patients per week etc. This information often provides useful information with regards to the health planning process (for instance the average number of rooms required over a period of time) however a weakness is that it is often not sensitive to variation during the time period. DES is an ideal tool to complement the health care planning process in that it can provide information on variance throughout the modeling process. DES can also be adapted to model specific patient routes (pathways) adding valuable insight to the health care planning process.

\subsection{Accident and Emergency (A\&E) and Urgent Care Centre (UCC) processes}

A\&Es (also known as emergency departments) are an area of interest to health care planners as they receive a range of patients requiring different services. Better analysis of patient groups and their pathways allows focusing of care towards particular patients. This focus can help to improve both health outcomes for the patient as well as reduce their length of stay in the facility. A\&E departments in the UK treat a wide range of patients, ranging from minor injuries to life threatening conditions and it forms a significant source of hospital admissions. Within a traditional A\&E model, patients often arrive into A\&E via two generic routes: Ambulance arrivals - where patients arrive via an ambulance; or Walk-in arrivals - nonambulance arrivals. On arrival into A\&E, patients are often triaged into categories for treatment by clinical staff. Table 1 illustrates triage code categories and their descriptions for the A\&E data source used for this study. The triage codes in Table 1 illustrates the wide range of patients that arrive in A\&E. Triage types range from immediate treatment (code 1) to non-urgent treatment (codes 5 and 6). After treatment in A\&E, patient are either discharged home or admitted into hospital. Treatment within A\&E is often supported by a range of activities including imaging and pathology.

Table 1: A\&E Triage codes by description, number of attendance and $\%$ of attendance

\begin{tabular}{|c|c|c|c|}
\hline Triage codes & Description & Number & $\%$ \\
\hline 1 & Immediate & 875 & 0.7 \\
\hline 2 & Very urgent & 11,860 & 9.7 \\
\hline 3 & Urgent & 38,626 & 31.5 \\
\hline 4 & Standard & 69,464 & 56.6 \\
\hline 5 & Non-urgent & 372 & 0.3 \\
\hline 6 & Non-urgent & 1,530 & 1.2 \\
\hline- & Total & 122,727 & 100.0 \\
\hline
\end{tabular}


Table 1 shows that $58 \%$ of patients require treatment for minor illness and injuries (codes 4 to 6 ) and $42 \%$ (code 1 to 3 ) of patients required care that is more urgent. Arguably, patients with minor injuries and illnesses might receive more effective treatment by General Practitioners (GPs) or Nurse Practitioners. New models of care suggest that conventional A\&E's may not be the most effective method of treating such a wide range of patients.

Alternative models of care suggest that emergency care might be delivered more efficiently by segregating major and minor patients. One alternative care model is to supplement an A\&E provision with an Urgent Care Centre (UCC). UCCs (supported by GPs and specialist nurses) are becoming increasingly common in the UK and they focus their care delivery towards attendees with minor injuries and illnesses leaving conventional A\&Es to focus their care delivery towards more seriously ill patients. Emergency Nurse Practitioners or Emergency Care Practitioners sometimes run UCCs. A multi-professional team including GPs, nurses and pharmacist usually supports Emergency Nurse Practitioner's. Often, especially in urban areas, UCCs are located adjacent to A\&E departments and act as the point of entry into the emergency department. There is evidence to show that when compared to A\&E clinicians, primary care physicians deliver efficient quality care. To further support the clinical case a study at the City Hospital, Birmingham, UK (Ansari et al. 2008) showed Primary care physicians saw more patients at lower cost compared to senior house officers with no difference recognized in the number of investigations. Reviewing Table 1, this study assumed that triage codes 4, 5 and 6 met the criteria of UCCs minor illness and minor injuries and triage code 1, 2 and 3 met the criteria for A\&E. As such, Table 1 was updated to show A\&E and UCC criteria and this is illustrated in Table 2.

Table 2: Table 1 A\&E triage codes re-assigned to A\&E and UCC codes

\begin{tabular}{|c|c|c|}
\hline Triage & Description & New model \\
\hline 1 & Immediate & A\&E \\
2 & Very urgent & A\&E \\
3 & Urgent & A\&E \\
4 & Standard & UCC \\
5 & Non-urgent & UCC \\
6 & Non-urgent & UCC \\
\hline
\end{tabular}

\section{MODEL DEVELOPMENT USING REAL DATA}

Increasingly, it is considered good practice to separate treatment pathways for adults and paediatrics to focus treatment and care to their requirements. As such, elderly and paediatric patients are sometimes considered special groups. Elderly patients are sometimes in a confused state and their range of illnesses often results in extended stays in emergency departments. In contrast, paediatric patients are often prioritized for treatment. Within this model, paediatric and elderly patients were defined as special cases and as such defined their own individual pathways. In the model, paediatric and elderly patients were defined by age: paediatric 16 years of age or under and elderly 75 years of age or older. The remaining adult group was divided into two distinct pathways dependent on acuity: Adult - A\&E; and Adult - UCC. Although resuscitation patients often require the most urgent attention, they formed a relatively low number of the overall attendances and consequently were not modeled in this study. A summary of the UCC patient groups (Pathways) derived from real data (1 year's activity) is shown in Table 3. Models were developed using the simulation software Simul8 working in conjunction with interactive input and output Excel worksheets. 
Table 3: UCC pathways (derived from A\&E data) showing number of patients and percentages. Resuscitation data included for completeness but was not modeled as a pathway.

\begin{tabular}{|c|c|c|}
\hline Pathway & Number of patients & Percentage (\%) \\
\hline Adult-A\&E & 31,641 & 25.8 \\
Adult-UCC & 47,135 & 34.4 \\
Elderly & 12,538 & 10.2 \\
Paediatric & 30,538 & 24.9 \\
Resuscitation & 875 & 0.7 \\
\hline Total & 122,727 & 100 \\
\hline
\end{tabular}

An overview of the model schematic is shown in Figure 1. The first step in the model (Pathway Arrivals) generated simulation icons in accordance with their pathway arrival pattern. Once generated, simulation icons passed though a queue bin before entry in to a simulation work centre to be processed. Queue bins acted as waiting areas (queuing area) if resources were unavailable at the work centre. In Figure 1, queue bins are shown as Pathway Queues. At the simulation work centres (shown as Pathway Process in Figure 1), patient icons were 'treated' in simulation time using average process times generated by the model. The average process time in this model only refers to direct doctor/nurse clinical treatment time. The treatment area might be a room or a cubicle and term 'room' in this study relates to either treatment area type. This model also assumed that resources (rooms) were fully staffed to treat simulation patients during the process time. Resources (shown as Pathway Resources in Figure 1) were assigned (if available) at the start of the simulation work centre process and released on completion. Exit from the simulation work centre completed the simulation process.

At each step in the simulation, time labels were attached to the simulation icons. These labels were used to calculate and store arrival times, queuing times and treatment times for all the simulation icons traveling on their modeled pathways. The same sub-routines were used by all the work centre process to $\log$ and store the time labels. This technique both optimized modular design (using verified code) and supported rapid development of new pathways.

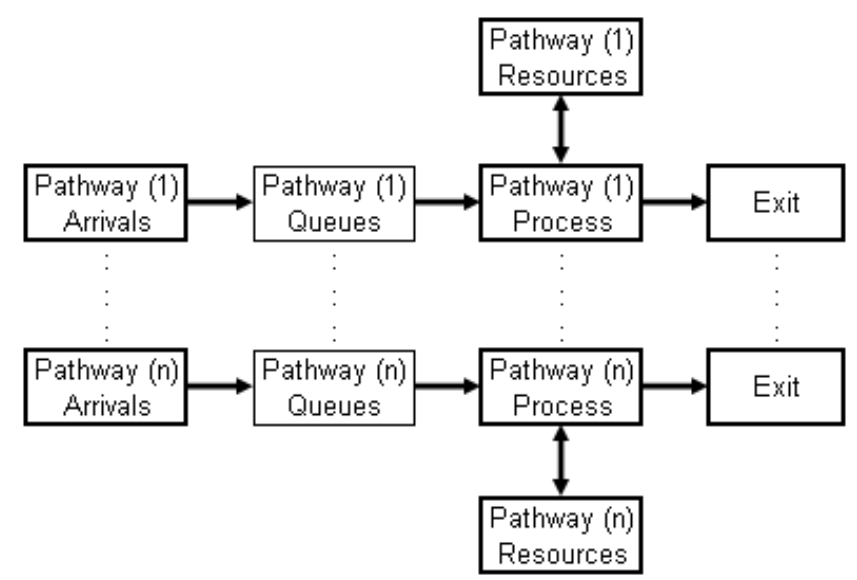

Figure 1: Schematic showing simulation model process.

Simulation data was recorded for 24 hours commencing at midnight after a 24-hour warm up period. A typical trial run was 50 simulations. The remainder of this section will show the staged model development and illustrate how real data was used to define arrival patterns, set room resources and provide evidence of model validation and verification between real and simulated data. 


\subsection{Extracted data to group patients by acuity and type and definition of their arrival patterns - Stage 1}

This sub-section will describe how real data was extracted to group patients into pathways and used to define their arrival patterns. Patient categories in the model were assigned pathways as shown in Table 3. Using the defined patient pathways, each pathway arrival profile was extracted from real A\&E data and this used to generate arrival profiles (Pathways Arrivals) in the simulation model. An example of the arrivals profile for adult-UCC is illustrated in Figure 2. Figure 2 shows the average arrival by hour over the data collection period of a year. In addition, Figure 2 shows the calculated percentage proportions split over 24 hours. The hourly percentage uses the arrivals per day (129.14) to calculate the patients per hour, which is used to calculate the inter arrival time. Using the adult-UCC example, 0 ArrivalHr (00:00 hrs to $00: 59 \mathrm{hrs}$ ) saw $2.29 \%$ of the activity over an average 24 hours and this equated to 2.96 patients per hour (129.14 patients per day $\times 2.29 \%)$. The inter arrival time $(20.30=60 / 2.96)$ was imported at the start of the mode run and updated hourly during the run cycle.

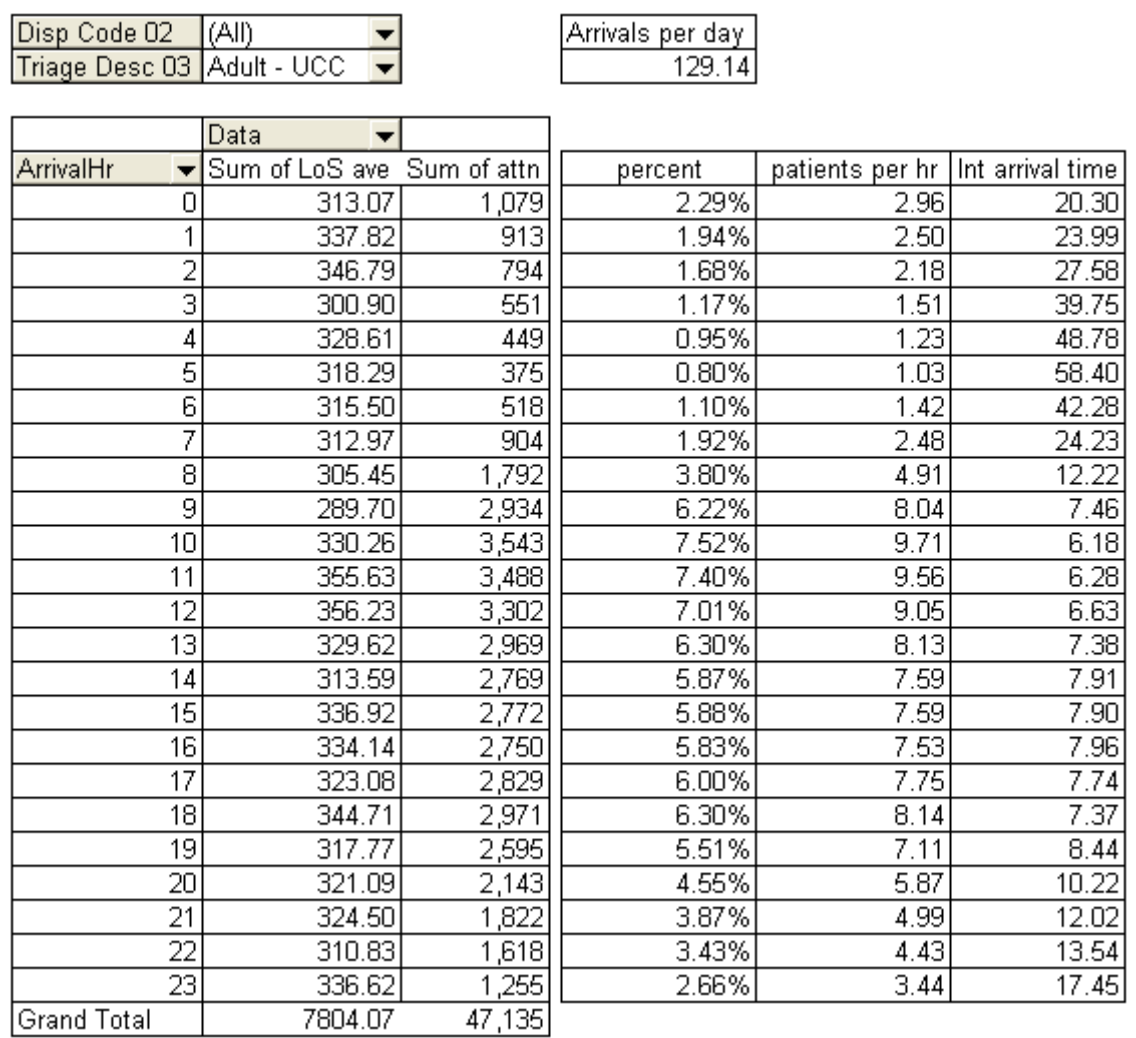

Figure 2: Adult - UCC arrival input example.

\subsection{Determine pathway room resources and process times using pathway activity - Stage 2}

This sub-section describes how room resources were determined in the model. The number of room resources modeled for each pathway was defined by calculations defined in Health Building Note (HBN) 11-01 (Facilities for primary and community care services). HBN 11-01 specifically related to primary and community services and as such is particularly suited to model UCCs. In contrast, HBN 11-01 although not specifically designed for emergency departments, it was used to model A\&E room (cubicle) requirements for this study. Using HBN 11-01 (with an occupancy of 80\% over 24 hours, 7 days a week) the initial number of adult-A\&E rooms were calculated as follows. Using 31,641 adult-A\&E attendances and assuming 100 minutes average process time: patient/weeks per year $=31,641 / 52=608$; appointment 
time $=608 *(100 / 60)=1,014 \mathrm{hrs} /$ week; room availability at occupancy $=24 * 7 * 0.8=134 \mathrm{hrs} /$ week; rooms $=1,014 / 134=8$ (rounded up). The other pathway rooms were calculated using the method described above and are shown in column 'Rooms' in Table 4.

Table 4: Pathway initial assumed process times and number of rooms. Note the process time assumes direct doctor/nurse clinical treatment.

\begin{tabular}{|c|c|c|}
\hline Pathway & Process time (mins) & Rooms \\
\hline Adult-A\&E & 100 & 8 \\
Adult-UCC & 40 & 5 \\
Elderly & 100 & 3 \\
Paediatric & 40 & 3 \\
\hline
\end{tabular}

The assumed process times (also shown in Table 4) reflected approximate times based on health planning experience with a number of Trust's. The process times shown in Table 4 represented a starting point in the development in the models. Process times were calibrated to best fit the actual data and this process is described in the next stage.

\subsection{Run and calibrate the model by comparing errors in length of stay between real and modeled pathways and adjusting process times if applicable - stage 3}

The average process (treatment) times shown in Table 4 represented a starting point to calibrate the model's length of stay to the actual length of stay. To calibrate the model, each pathway the model ran a range of process times to compare the modeled length of stay against the actual length of stay. Specifically, for each pathway, the cumulative distribution of the actual length of stay was compared with the cumulative distribution of the modeled length of stay for a range of process times. The cumulative distribution is shown by:

$$
F(x 1, x 2)=\sum_{t \leq x 1, x 2}^{0.95} f(t), \text { where }
$$

$F(x 1)$ is the cumulative distribution for actual length of stay, $F\left(x_{2}\right)$ is the cumulative distribution for the modeled length of stay and $f(t)$ in steps in 15 minutes up to $95 \%$ of the cumulative distribution of the actual length of stay. The process times (rounded to the nearest whole number) with the minimum errors between actual and modeled length of stay $\left(\bar{d}=F(x 1)-F\left(x_{2}\right)\right)$ is shown in the adjusted column Table 5. These adjusted process times were in effect used to calibrate the model and used to validate and verify the model. By observation, Table 5 suggested the similarity between the initial and the adjusted clinical treatment time supported the estimates of clinical process time for the chosen pathways.

Table 5: Pathway initial and adjusted pathway process times. Note the process time assumes direct doctor/nurse clinical treatment.

\begin{tabular}{|c|c|c|}
\hline \multirow{2}{*}{ Pathway } & \multicolumn{2}{|c|}{ Process time (minutes) } \\
\cline { 2 - 3 } & Initial & Adjusted \\
\hline Adult-A\&E & 100 & 110 \\
Adult-UCC & 40 & 42 \\
Elderly & 100 & 92 \\
Paediatric & 40 & 35 \\
\hline
\end{tabular}




\subsection{Model validation and verification - stage 4}

Input-output validation techniques were applied to both pathway arrivals and pathway lengths of stays to validate and verify the model. Using pathway arrivals and the methodology described by Banks et al. (2000), systems outputs (real data) $Z_{j}$ were compared with modeled outputs $W_{j}$ where $j=$ the hour of the day 1 to $K$ (1 to 24$), d_{j}=Z_{j}-W_{j}$ and;

$$
\begin{gathered}
\sigma_{d}^{2}=S_{d}^{2}=\frac{1}{K-1} \sum_{j=1}^{K}\left(d_{j}-\bar{d}\right)^{2} \\
\bar{d}=\frac{1}{K} \sum_{j=1}^{K} d_{j}
\end{gathered}
$$

Using the paired $t$-test, the null hypothesis was represented by $d_{j}=Z_{j}-W_{j}=\mu_{d}=0$ and tested against the alternative hypothesis of $d_{j}=Z_{j}-W_{j}=\mu_{d} \neq 0$, where:

$$
t_{0}=\frac{\bar{d}-\mu_{d}}{S_{d} / \sqrt{K}}
$$

\subsubsection{Arrivals}

Testing the t-statistic for pathway arrivals concluded acceptance (at a 95\% confidence level) of the null hypothesis for all pathways. As such, this test provided evidence that modeled data effectively matched real data and could act as an effective arrival generator for pathways in the model. As an example, real and modeled arrival profiles for adult - UCC (averaged over 24-hour period) is illustrated in Figure 3.

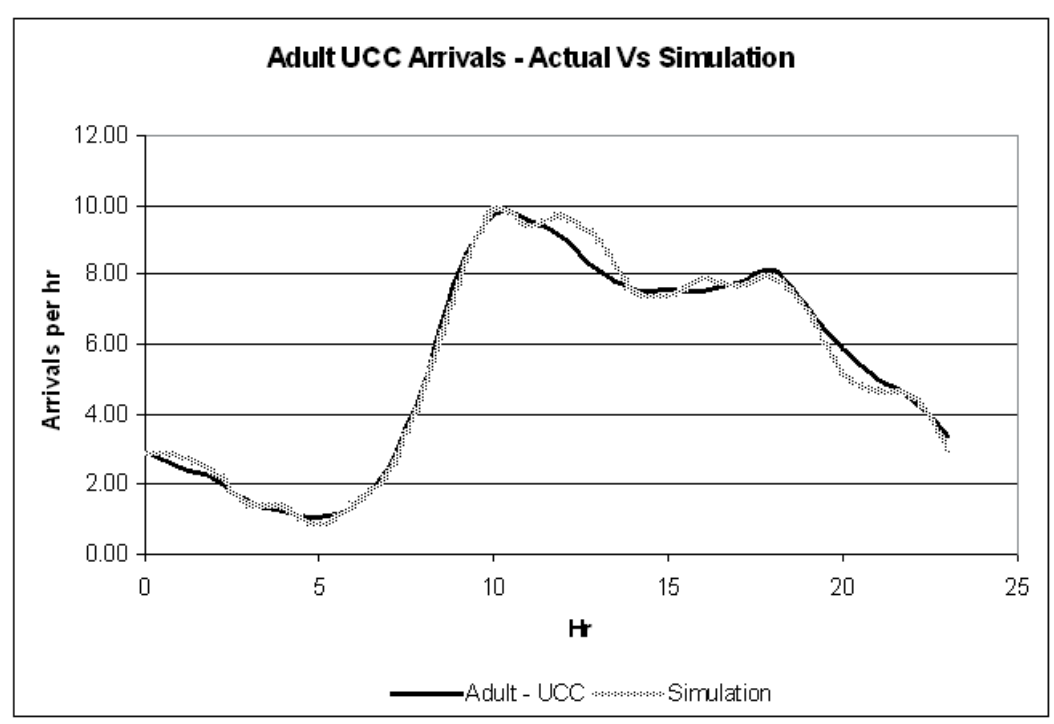

Figure 3: Average actual (real) arrivals versus average simulation arrivals for adult-UCC. 
Virtue, Chaussalet, and Kelly

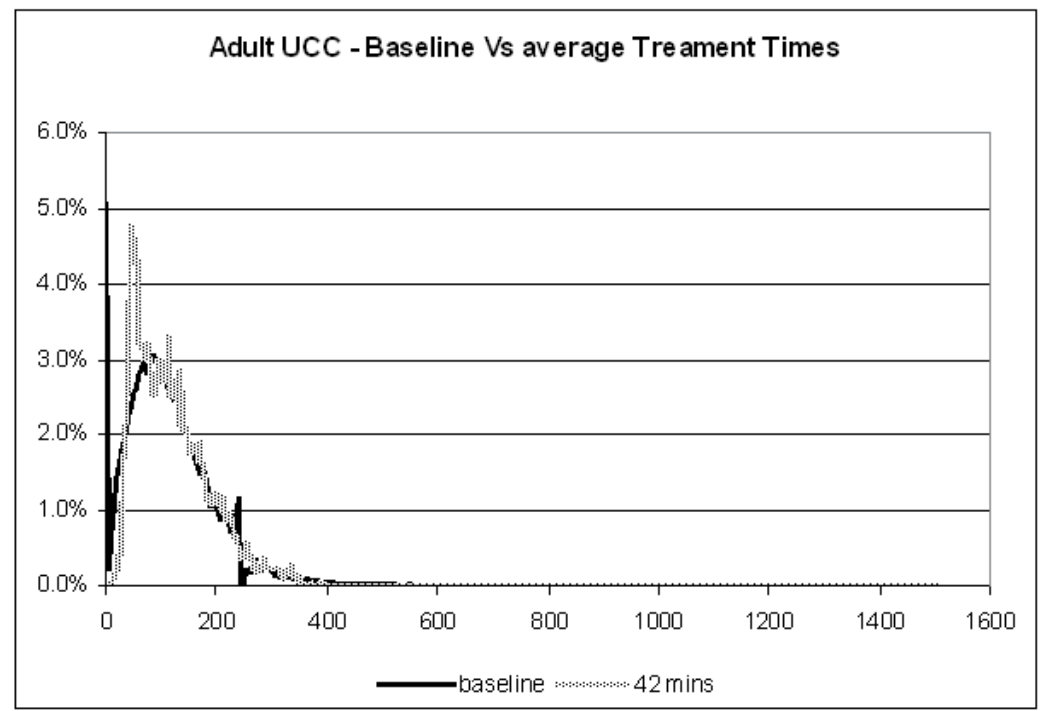

Figure 4: Actual (real) length of stay actual versus simulated length of stay for adult-UCC.

\subsubsection{Length of stay}

Paired $\mathrm{Z}$ tests were performed on pathway lengths of stay where as above, real data $Z_{j}$ was compared with modeled data $W_{j}$, where $j=1$ to $K$ was $95 \%$ of the real length of stay in steps of 15 minutes. Testing the Z-statistic for pathway lengths of stay concluded acceptance (at a 95\% confidence level) of the null hypothesis $\left(d_{j}=Z_{j}-W_{j}=\mu_{d}=0\right)$ for all pathways. As an example, real and modeled arrival profiles for adult - UCC is illustrated in Figure 4. Figure 5 illustrates average and median lengths of stay for pathways and overall (all pathways). Paired $t$ tests were also performed on average and median data for pathways and all (the combined data) to compare real and modeled data as shown Figure 5. Test results here also concluded (at a 95\% confidence level) acceptance of the null hypothesis for all pathways and therefore reinforced evidence above of good correlation between the real lengths of stay and the modeled length of stay for pathways. As such, the paired test provided evidence that modeled outputs reflected real outputs and as such validated the model. This validation also suggested an adequate level of model coding (verification) within the parameters of the developed model.

\begin{tabular}{|l|c|c|c|c|}
\hline \multicolumn{3}{|c|}{ Length of Stay Comparisons (minutes) } \\
\hline \multirow{2}{*}{ Pathways } & \multicolumn{2}{|c|}{ Actual } & \multicolumn{2}{c|}{ Simulation model } \\
\cline { 2 - 5 } & Average & Median & Average & Median \\
\hline Adult - A\&E & 168 & 152 & 162 & 148 \\
\hline Adult - UCC & 113 & 101 & 115 & 102 \\
\hline Elderly & 192 & 174 & 191 & 163 \\
\hline Paediatric & 113 & 102 & 111 & 92 \\
\hline All & 135 & 119 & 134 & 120 \\
\hline
\end{tabular}

Figure 5: Length of stay comparisons between actual (real) and simulated.

\section{FINDINGS}

This paper has shown how average simulation process times can act as estimators for real length of stay and provide pathway information in a health care setting. This paper has shown too that a simplified model could be used to help reconfigure emergency care services and provide modeling insights into those services. The model showed pathways lengths of stay, modeled using average process (treatment) times, 
driven by real arrival data with HBN calculated resources, matched real pathway lengths of stay within a 95\% confidence level. Simplified models directly linked to real data and with reduced model development time, allow stakeholders to open up the black box and better appreciate high-level interactions in their systems. This opens up the model to a number of arenas including; testing parameters such as queues during run time to determine when might they appear in the cycle, how big those queues might get and how long might they last?; are queues pathway specific; what impact does changing resources have; and what impact does changing process time have? For illustration, the adult-UCC queue output is shown in Figure 6. The model also suggests similarities in the direct clinical treatment time used and the pre model assumptions. The findings however do appear to show a difference between the average clinical time (process time) used to drive the work centre and the overall length of stay. For example, adult-UCC used an average process time of 42 minutes and this resulted in a modeled average time of 115 minutes (or median time of 102 minutes) against a real world average of 113 minutes (or real world median of 101 minutes). One area to investigate would be the resources allocated. In this model (and often in the real world) resources are calculated using activity averaged over a period. We can see from the arrival graphs that arrivals (reference Figure 3) increases mid morning, stays quite high in the day before dropping in the evening. Similarly, we can see queues starting to increase at around mid morning - see Figure 6 before dropping of in the late evening. In this example, variable resources (high in the day, low at night) could be an area for further investigation. The ability to model patients pathways in a shorten development time using simple, transparent models attempts to overcome objections to black box models as described by Sinreich and Marmor. Modeling assumptions could be quickly modified and the model re-run. For example, in the light of current NHS fiscal constraints, simplified models could be used to explore the impact of fewer resources on pathways and the impact on their length of stay. The models developed here were particularly focused towards addressing real world links, the needs of stake-holders and to be used as a decision making tool as discussed by Taylor et al., Günal and Pidd and Sanchez et al. The findings from these simplified models could well be a pre cursor to more detailed pathway studies and models that are more complicated

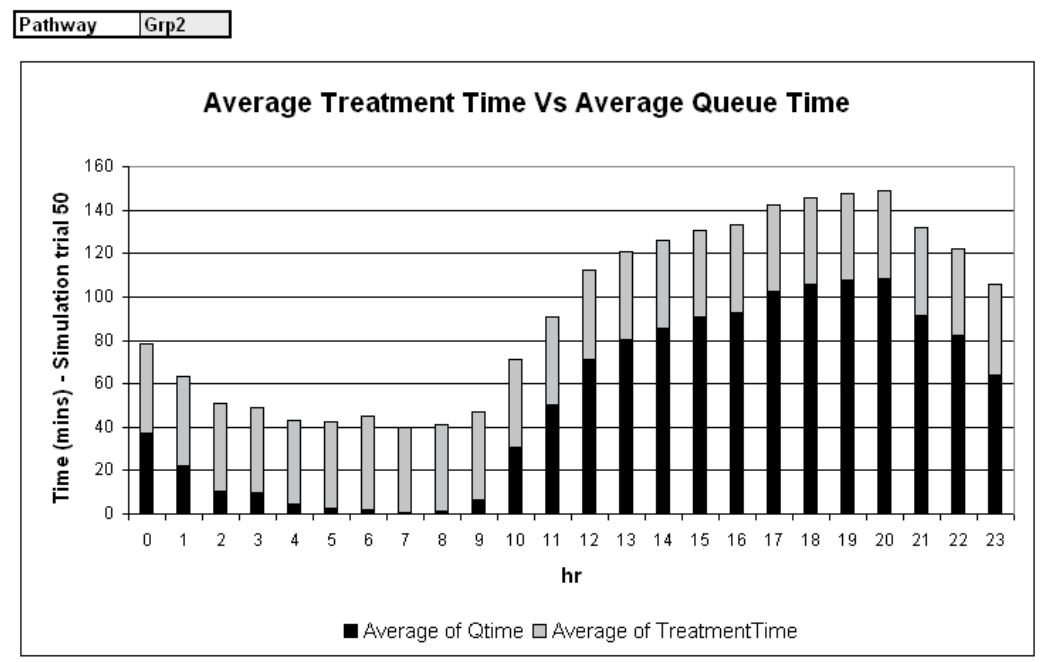

Figure 6: Adult-UCC Modeled Queues

\section{LIMITATIONS AND FURTHER WORK}

This study represents an early stage of development and work continues to further develop the model. Work is continuing to better understand the differences in the real and modeled worlds between the average treatment time and the average length of stay. Other areas for development include models to assess the impact of other variables such as day of week, month of year and the impact of patient mix. The models discussed in this paper were developed in a modular format with a mind to support rapid model devel- 
opment to model patient pathways in other health care settings. This too is an area of future work. The example above discussed fixed resources with changing input. Changing resources throughout the day is another area of model development.

\section{REFERENCES}

Ansari, Z., A. Saha, H. Azra, P. John, and P. Ahee. 2008. "Urgent care Centres: A new development in the United Kingdom." Annals of Emergency Medicine 51:550.

Banks, J., J. S. Carson, B. L. Nelson, and D. M. Nicol. 2000. Discrete-Event System Simulation. 3rd ed. Upper Saddle River, New Jersey: Prentice-Hall, Inc.

Barnes, C., J. Quiason, C. Benson, and D. McGuiness. 1997. "Success stories in simulation in health care." In Proceedings of the 1997 Winter Simulation Conference, edited by S. Andradóttir, K. J. Healy, D. H. Withers, and B. L. Nelson, 1280-1285. Piscataway, New Jersey: Institute of Electrical and Electronics Engineers, Inc.

Bowers, J., M. Ghattas, and G. Mould. 2009. "Success and failure in the simulation of an Accident and Emergency department." Journal of Simulation 3:171-178.

Ceglowski, R., L. Churilov, and J.Wasserthiel. 2007. "Combining data mining and discrete event simulation for a value-added view of a hospital emergency department." Journal of the Operational Research Society 58:246-254.

Codrington-Virtue, A., T. Chaussalet, P. Millard, P. Whittlestone, and J. Kelly. 2006. "A system for patient management based discrete-event simulation and hierarchical clustering." 800-804. In Proceedings of the 19th IEEE International Symposium on Computer-Based Medical Systems.

Codrington-Virtue, A., P. Whittlestone, J. Kelly, and T. Chaussalet. 2005a. "Developing an application of an accident and emergency patient Simulation modeling using an interactive framework." In Proceedings of the 31st Annual Meeting of the EURO Working Group on OR Applied to Health Services.

Codrington-Virtue, A., P. Whittlestone, J. Kelly, and T. Chaussalet. 2005b. "An interactive framework for developing simulation models of hospital accident and emergency services." 114: 277-283. Medical and Care Compunetics 2.

El-Darzi, E., C. Vasilakis, T. Chaussalet, and P. Millard. 1998. "A simulation modelling approach to evaluating length of stay, occupancy, emptiness and bed blocking in a hospital geriatric department." Health Care Management Science 1:143-149.

Fletcher, A., D. Halsall, S. Huxham, and D. Worthington. 2007. "The DH accident and emergency department model: a national generic model used locally." Journal of the Operational Research Society 58:1554-1562.

Griffiths, J., N. Price-Lloyd, M. Smithies, and J. Williams. 2005. "Modelling the requirement for supplementary nurses in an intensive care unit." Journal of the Operational Research Society 56:126-133.

Günal, M., and M. Pidd. 2006. "Understanding accident and emergency department performance using simulation." In Proceedings of the 2006 Winter Simulation Conference, edited by L. R. Perrone, F. P. Wieland, J. Liu, B. G. Lawson, D. M. Nicol, and R. M. Fujimoto, 446-452. Piscataway, New Jersey: Institute of Electrical and Electronics Engineers, Inc.

Günal, M., and M. Pidd. 2010. "Discrete event simulation for performance modeling in health care." Journal of Simulation 4:42-51.

Harper, P., and M. Pitt. 2004. "On the challenges of healthcare modeling and a proposed project life cycle for successful implementation." Journal of the Operational Research Society 55:657-661.

Katsaliaki, K., and S. Brailsford. 2007. "Using simulation to improve the blood supply chain." Journal of the Operational Research Society 58:219-227.

Proudlove, N., S. Black, and A. Fletcher. 2007. "OR and the challenge to improve the NHS: modelling for insight and improvement in in-patient flows." Journal of the Operational Research Society 58:145-158. 
Rauner, M., S. Brailsford, and S. Flessa. 2005. "Use of discrete-event simulation to evaluate strategies for the prevention of mother-to-child transmission of HIV in developing countries." Journal of the Operational Research Society 56:222-233.

Sanchez, S., D. Ferrin, T. Ogazon, J. Sepulreda, and T. Ward. 2004. "Emerging issues in healthcare simulation". In Proceedings of the 2000 Winter Simulation Conference, edited by J. A. Joines, R. R. Barton, K. Kang, and P. A. Fishwick, 1999-2003. Piscataway, New Jersey: Institute of Electrical and Electronics Engineers, Inc.

Sinreich, D., and Y. Marmor. 2004. "A simple and intuitive simulation tool for analyzing the performance of emergency departments." In Proceedings of the 2004 Winter Simulation Conference, edited by R. G. Ingalls, M. D. Rossetti, J. S. Smith, and B. A. Peters, 1994-2002. Piscataway, New Jersey: Institute of Electrical and Electronics Engineers, Inc.

Taylor, S., T. Eldabi, G. Riley, R. Paul, and M. Pidd. 2009. "Simulation modelling is 50! Do we need a reality check?" Journal of the Operational Research Society 60:569-582.

Vasilakis, C., and A. Marshall. 2005. "Modelling nationwide hospital length of stay: opening the black box." Journal of the Operational Research Society 56:862-869.

\section{AUTHOR BIOGRAPHIES}

ANTHONY VIRTUE is a part-time PhD student within the Health and Social Care Modeling Group (HSCMG) at the University of Westminster and his primary interest is the application of discrete-event simulation modeling techniques to healthcare services and systems. Anthony works full-time and is employed as a Senior Consultant within EC Harris LLP applying healthcare modeling techniques within their Strategy and Transformation Group. Anthony obtained a BEng in Mechanical Engineering from Brunel University, an MSc in Engineering Management from Bristol University and an MSc in Decision Sciences from the University of Westminster. His email address is anthony.virtue@echarris.com

JOHN KELLY was a founding Director of RKW Healthcare Consultants and is now a Partner with EC Harris LLP within its Strategy and Transformation Group. His background is in sociology and business strategy and he has 30 years' experience in the health sector. John has a special interest in healthcare futures and has presented at major conferences in the UK and overseas. He also has extensive experience in developing innovative Models of Care for acute/community and mental health services and in securing the support of key staff to strategic change through workshops and seminars. John has been involved in a wide variety of strategic/service and estate reviews and briefing/evaluation studies covering major acute and mental health Trusts. He is also the author of key guidance documents for the planning of health facilities based on DoH research projects and consultancy work for the NHS and private sector. His email address is john.kelly@echarris.com

THIERRY CHAUSSALET is currently Professor of Healthcare Modelling at the University of Westminster, which he joined after studying in France and the USA. His research interests lie in the development and use of informatics and modelling techniques to improve healthcare management. He serves currently on the Editorial Board of various healthcare modelling and informatics journals. He is member of the EPSRC Peer Review College, Chair of the Health and Social Services Study Group and member of the Council of the Operational Research Society. Active promoter of the use of modelling in the health services, he has been member of the Steering Group of MASHnet, the UK healthcare modeling and simulation network, since 2005, and of the recently established Cumberland Initiative, a consortia of over 15 UK universities developing a centre for system modelling and service science in health care. His email address is chausst@westminster.ac.uk 